\title{
Library versus Library Recognition and Inhibition of the HIV-1 Nef Allelome
}

Allison Olszewski ${ }^{\dagger}$ and Gregory A. Weiss ${ }^{\dagger \neq *}$

Departments of Chemistry, ${ }^{\ddagger}$ Molecular Biology and Biochemistry,

University of California, Irvine, California 92697-2025

Supporting Information

(a)

$$
\begin{array}{ccc}
\text { Compound } & & \text { \% Nef-p53 inhibition } \\
& \text { wild-type } & \\
\mathbf{1} & & 101.1 \pm 2.3 \\
\mathbf{2} & & 96.8 \pm 0.9 \\
\mathbf{3} & & 94.7 \pm 1.0 \\
\mathbf{4} & & 93.9 \pm 1.0 \\
\mathbf{5} & & 68.5 \pm 3.0 \\
\mathbf{6} & & 19.1 \pm 9.5 \\
\mathbf{7} & & 11.9 \pm 6.1 \\
\mathbf{8} & & (-) 0.7 \pm 10.8 \\
& & \\
\mathbf{1} & \text { allelome } & \\
\mathbf{2} & & 53.8 \pm 1.4 \\
\mathbf{3} & & 93.3 \pm 1.1 \\
\mathbf{4} & & 25.7 \pm 3.2 \\
\mathbf{5} & & 93.3 \pm 0.7 \\
\mathbf{6} & & 60.9 \pm 2.4 \\
\mathbf{7} & & 56.1 \pm 0.4 \\
\mathbf{8} & & 27.2 \pm 3.4 \\
& & 13.9 \pm 4.5
\end{array}
$$

(b)

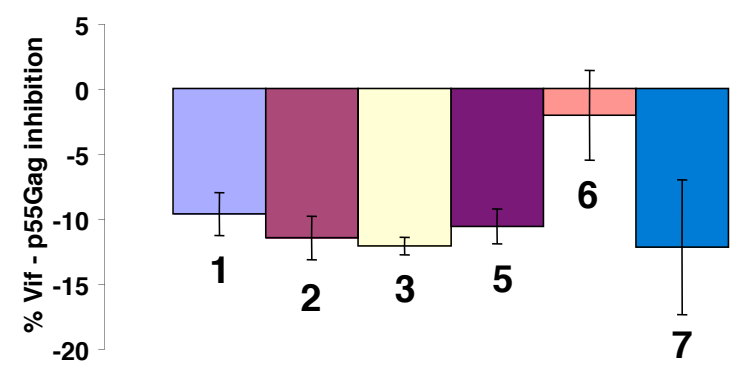

(c)

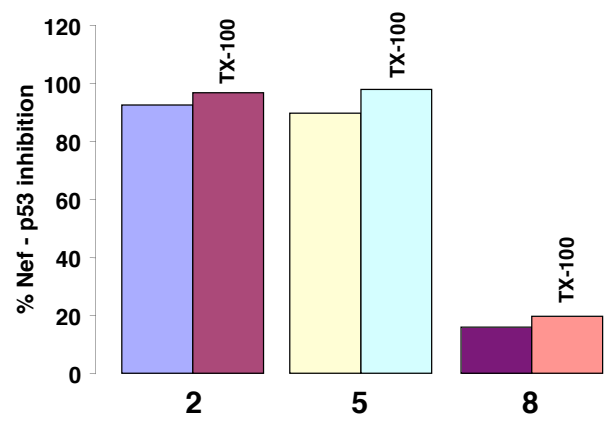

Figure S1. Small molecule competition ELISAs. (a) Inhibition of the Nef-p53 interaction. (b) Control assay for inhibition of the HIV-1 Vif - p55Gag interaction. This experiment demonstrates the reported Nef inhibitors do not block the anti-M13 phage antibody, nor inhibit HRP activity. (c) Control assay for inhibition of the wt Nef-p53 interaction in the absence or presence of Triton X-100 (TX-100). 
Oligonucleotides. DNA degeneracies are represented by the IUB code $(K=G / T . M=A / C, N=$ $\mathrm{A} / \mathrm{C} / \mathrm{G} / \mathrm{T}, \mathrm{R}=\mathrm{A} / \mathrm{G}, \mathrm{S}=\mathrm{G} / \mathrm{C}, \mathrm{W}=\mathrm{A} / \mathrm{T}, \mathrm{Y}=\mathrm{C} / \mathrm{T})$. StopNef11-24, 5'-ATT GGA TGG CCT GCT GTA TGA TGA AGA AGT AGA CGA GCT GAG-3'; StopNef41-54, 5'-GGA GCA ATC ACA AGT AGC TGA TGA GCA GCT AAC AAT GCT GC-3'; Nef2-30, 5'-GGT GGC AAG TGG TCA AAA HSK MGT GTG RKT GGA TGG BCT RCT RTA AGG GAA AGA ATK MRR CVA RYT GAG CCA GCA GCA GAT GGG GTG-3'; Nef31-63, 5'-GGA GCA GTA TCT CGA GAC CTA GVM ARA YAY GGA GCA VTC ACA AGT AGC AAT ACA SCA RCT AMC AAT GCT GMT TGT GCC TGG STA GAA GCA CAA GAG GAG GAA G-3'.

Compounds. Libraries of batzelladine and crambescidin analogs were previously described ${ }^{1-6}$. Each compound was stored at $-20{ }^{\circ} \mathrm{C}$ at an average concentration of $10 \mathrm{mg} / \mathrm{ml}$ in DMSO.

Construction of phage-displayed Nef N-terminal library. The Nef phagemid ${ }^{1}$ was used as a template for library construction. The Nef N-terminal library fused to the P8 major coat protein of M13 phage was constructed using a previously described method ${ }^{7}$. The phagemid stop template was generated by oligonucleotide-directed mutagenesis, which introduced stop codons into the N-terminus at residues 17 , 18, 47, and 48 with oligonucleotides NefStop11-24 and NefStop41-54. The resulting template was used to introduce the desired mutations by the same mutagenesis technique using oligonucleotides Nef2-30 and Nef31-63. The theoretical diversity of the library was $6.4 \times 10^{7}$ and the experimental diversity was $2.5 \times 10^{7}$. The library was stored at $-78{ }^{\circ} \mathrm{C}$ in $10 \%$ glycerol for later use.

Phage selection. Phage from the Nef N-terminal library were cycled through multiple rounds of binding selection with Nef ligands, such as p53 described here. Specific wells of a 96-well Maxisorp immunoplate were coated with p53 (Santa Cruz Biotechnology) (100 $\mu 1,5 \mu \mathrm{g} / \mathrm{ml}$ ) in 50mM carbonate buffer, $\mathrm{pH}$ 9.6) for $2 \mathrm{~h}$ at room temperature. The plate was then blocked for $30 \mathrm{~min}$ with ovalbumin $(0.2 \%)$ in PBS or BSA $(0.2 \%)$. For the first round of selection, the plate was washed five times with PT buffer $(0.05 \%$ Tween 20 in PBS). Separately, library phage was diluted 1:1 in POT (PBS, 0.2\% ovalbumin, $0.05 \%$ Tween 20 ) or PBT (PBS, 0.2\% BSA, 0.05\% Tween 20). The library phage solution was added directly to the corresponding well of target-coated assay plate. The plate was shaken for $2 \mathrm{~h}$ at room temperature and washed five times with PT buffer. Phage were eluted by addition of $\mathrm{HCl}(100$ $\mu 1,100 \mathrm{nM}$ ) and shaken vigorously for $5 \mathrm{~min}$. The eluted phage was immediately neutralized with tris(hydroxymethyl)aminomethane- $\mathrm{HCl}(33 \mu \mathrm{l}, 1.0 \mathrm{M}, \mathrm{pH} 8.0)$. PBS (100 $\mu \mathrm{l})$. The selection was then repeated using identical conditions, except the number of wash steps was increased by two and the blocking agent for even numbered selection rounds was BSA.

p53-Nef phage binding ELISA. Cultures of E. coli harboring individual phagemids were grown overnight in 96-well format at $37^{\circ} \mathrm{C}$ in 2 YT medium $(600 \mu \mathrm{l})$, carbenicillin $(50 \mu \mathrm{g} / \mathrm{ml})$, and M13-K07 helper phage $\left(10^{10} \mathrm{phage} / \mathrm{ml}\right)$. Cells were removed by centrifugation $(10 \mathrm{~min}, 12,000 \mathrm{x} g)$, and the supernatant was used directly in the phage ELISA. Specific wells of a Maxisorp immunoplate (96-well) were coated with p53 (Santa Cruz Biotechnology, Santa Cruz, CA (100 $\mu \mathrm{l}, 5 \mu \mathrm{g} / \mathrm{ml}$ ) in $50 \mathrm{mM}$ carbonate buffer, $\mathrm{pH}$ 9.6) for two hr at room temperature. The plate was then blocked for 30 min with ovalbumin (0.2\%) in PBS and washed five times with PT buffer (0.05\% Tween 20 in PBS). The phage culture supernatant solution was added directly to the corresponding wells of target-coated assay plates. The assay plates were shaken for $1 \mathrm{hr}$ at room temperature, and washed five times with PT buffer. After washing, plates were incubated with anti-M13/horseradish peroxidase (HRP) conjugate (100 $\mu 1,1: 5000$, Amersham Biosciences, Piscataway, NJ) in POT for $30 \mathrm{~min}$, and washed eight times with PT buffer and twice with PBS. Plates were developed with o-phenylenediamine dihydrochloride $/ \mathrm{H}_{2} \mathrm{O}_{2}$ solution $(100 \mu$, $1 \mathrm{mg} / \mathrm{ml} \mathrm{OPD}$ and $0.02 \% \mathrm{H}_{2} \mathrm{O}_{2}$ ) in citric acid buffer (50 mM citric acid, $50 \mathrm{mM} \mathrm{Na} \mathrm{HPO}_{4}, \mathrm{pH} 5.0$ ). After 
ten minutes, the absorbance was measured spectrophotometrically at $450 \mathrm{~nm}$ using a 96-well microtiter plate reader ( $\mu$ Quant, Bio-Tek). Culture supernatant from p53 binding selectants was subjected to DNA sequencing.

Small-molecule competition ELISA. Inhibition of the phage-displayed Nef N-terminal library was demonstrated as described for inhibition of wild-type $\mathrm{Nef}^{1}$ with the following exception. The Nef allelome phage concentration was $13.3 \mathrm{nM}$. Inhibition data are shown in Fig. S1a and graphically represented in Fig. 2. As a control, the compounds described in Fig. 2 were assayed for inhibition of the phage-displayed HIV-1 Vif - p55Gag interaction (Fig. S1b). Assay conditions were similar to the Nefp53 assay with the following exceptions. The blocking agent was $0.2 \%$ BSA in PBS, and the experiment was performed in duplicate with error bars indicating standard error. Fig. S1c demonstrates that the addition of Triton X-100 has no effect on the observed Nef inhibition. An inhibition ELISA was performed as described for the wt Nef - p53 interaction (unlabeled columns in Fig. S1c). Columns labeled TX-100 in Fig. S1c were treated identically with the following exception. Triton X-100 (final concentration of $0.01 \%$ ) was added to the wt Nef phage and compound solution. The solution was incubated for one hour prior to incubation on p53-coated Maxisorp plate as usual.

Table S1. Design of the Nef allelome.

\begin{tabular}{|c|c|c|c|c|c|c|c|c|}
\hline \multirow[b]{2}{*}{ Position } & \multirow[b]{2}{*}{ wild-type } & \multicolumn{2}{|c|}{ Progression } & \multirow[b]{2}{*}{ mutations } & \multirow[b]{2}{*}{ codon } & \multicolumn{3}{|c|}{$\%$ rapid progression } \\
\hline & & Non & Rapid & & & p53 & actin & $\mathrm{p} 56^{\text {lck }}$ \\
\hline 8 & $\mathrm{~s}$ & & & $\mathrm{~S}, \mathrm{R}, \mathrm{C}, \mathrm{W}, \mathrm{P}, \mathrm{T}$ & hsk & & & \\
\hline 9 & $\mathrm{~s}$ & & & $\mathrm{~S}, \mathrm{R}$ & mgt & & & \\
\hline 11 & $\mathrm{I}$ & & & $\mathrm{I}, \mathrm{V}, \mathrm{G}, \mathrm{S}$ & rkt & & & \\
\hline 14 & $P$ & & & $\mathrm{P}, \mathrm{S}, \mathrm{A}$ & bct & & & \\
\hline $15^{*}$ & A & $T$ & A & $A, T$ & rct & 85 & 70 & 67 \\
\hline 16 & $\mathrm{~V}$ & & & $\mathrm{~V}, \mathrm{I}$ & rta & & & \\
\hline 20 & M & & & $\mathrm{M}, \mathrm{I}$ & atk & & & \\
\hline 21 & $\mathrm{R}$ & & & $\mathrm{R}, \mathrm{K}, \mathrm{Q}$ & $\mathrm{mrr}$ & & & \\
\hline 22 & $\mathrm{R}$ & & & $R, P, Q$ & cva & & & \\
\hline 23 & A & & & $\mathrm{A}, \mathrm{T}, \mathrm{V}, \mathrm{I}$ & ryt & & & \\
\hline 38 & $\mathrm{E}$ & & & $E, A, D, G$ & gvm & & & \\
\hline $39^{*}$ & $\mathrm{~K}$ & K & $R$ & $\mathrm{~K}, \mathrm{R}$ & ara & 69 & 53 & 58 \\
\hline 40 & $\mathrm{H}$ & & & $\mathrm{H}, \mathrm{Y}$ & yay & & & \\
\hline 43 & $\mathrm{I}$ & & & $\mathrm{I}, \mathrm{L}, \mathrm{V}$ & vtc & & & \\
\hline 49 & A & & & $A, P$ & sca & & & \\
\hline 50 & A & & & $\mathrm{A}, \mathrm{T}$ & rct & & & \\
\hline $51^{*}$ & $\mathrm{~N}$ & $\mathrm{~N}$ & $T$ & $\mathrm{~N}, \mathrm{~T}$ & amc & 77 & 50 & 29 \\
\hline 54 & A & & & $A, D$ & gmt & & & \\
\hline 58 & $\mathrm{~L}$ & & & $L, V$ & sta & & & \\
\hline
\end{tabular}

Nef allelome design (Table S1). Positions associated with clinical HIV progression and nonprogression are denoted with a*. Nef mutations, based upon sequences isolated from the clinic, are encoded by codons (lowercase DNA bases in IUB code). Percent rapid progression denotes the percentage of Nef allelome sequences having the substitution pattern associated with rapid progression to AIDS.

\section{Sequences of Nef mutants (residues 2-60) selected from the Nef allelome.}

Nef mutants selected for binding to p53

\footnotetext{
Trial\# 1

P2: SGGKWSKWSVGGWPTIRERIRRTEPAADGVGAVSRDLGRHGAITSSNTATNNAACAWVEA

P4: SGGKWSKWSVGGWAAIRERIRRTEPAADGVGAVSRDLGRYGAVTSSNTATTNADCAWVEA

P6: SGGKWSKWRVGGWSAVRERMRPTEPAADGVGAVSRDLGRYGAVTSSNTAATNAACAWLKA

P7: SGGKWSKWRVSGWAAVRERMRNTEPAADGVGAVSRDLAKHGAVTSSNTPANNADCAWVEA

P8: SGGKWSKSRVIGWSAIRERMKRAEPAADGVGAVSRDLARYGALTSSNTAGNNAACAWVEA

P14 : SGGKWSKWSVIGWPAVRERMRNVKPAADGVGAVSRDLGRYGAITSSNTPGTNADCAWLEA

P15: SGGKWSKPRVSGWSAVRERMRPTAPAADGVGAVSRDLGKYGAVTSSNTPGTNADCAWVEA
} 
P1 6: SGGKWSKWSVVGWSAVRERIRRVEPAADGVGAVSRDLAKHGAVTSTNTAGTNAACAWVEA P17: SGGKWSKSSVSGWSAIRERMRRTEPAADGVGAVSRDLDKHGAVTSSNTAGTNAACAWVEA P2 0 : SGGKWSKRSVGGWATVRERMRRAEPAADGVGAVSRDLGRYGAVTSSNTATTNAACAWLEA P21: SGGKWSKWRVSGWSAIRERIRQTEPAADGVGAVSRDLARHGAITSSNTPATNADCAWLEA P22: SGGKWSKWRVGGWAAVRERMRQAEPAADGVGAVSRDLGRYGALTSSNTPATNAACAWLEA P25: SGGKWSKPRVGGWAAIRERMRQTEPAADGVGAVSRDLGRYGAITSSNTPATNAACAWLEA

Trial \#2

P1: GGKWSKPRVSGWAAIRERMRPVEPAADGVGAVSRDLGRHGAITSSNTAANNAACAWLEA P2: GGKWSKTRVGGWAAIRERMRPTEPAADGVGAVSRDLAKYGAITSSNTAANNADCAWVEA P3: GGKWSKPRVSGWSAVRERMRPAEPAADGVGAVSRDLDRHGAITSSNTPTNNAACAWVEA P4: GGKWSKWSVSGWPTIRERIRRAEPAADGVGAVSRDLGRYGAVTSSNTATTNAACAWVEA P5: GGKWSKPSVIGWPTVRERMRRTEPAADGVGAVSRDLGRYGALTSSNTPTNNAACAWVEA P7: GGKWSKTRVIGWAAVRERMRQVEPAADGVGAVSGDLGKHGAVTSSNTPTTNAACAWLEA P8: GGKWSKWRVSGWPTIRERMKRVEPAADGVGAVSRDLARYGAVTSSNTAANNAACAWLEA P10: GGKWSKPRVVGWAAIRERMRQVEPAADGVGAVSRDLGRHGAVTS SNTAATNAACAWLEA P11: GGKWSKWRVGGWSAVRERMQRVEPAADGVGAVSRDLGRYGALTSSNTPTTNADCAWLEA P13: GGKWSKRRVGGWSTVRERIKPAEPAADGVGAVSRDLARHGAVTSSNTPANNADCAWVEA P14: GGKWSKPRVSGWSAIRERMKPAEPAADGVGAVSRDLGRYGAVTSSNTATNNADCAWVEA P15: GGKWSKRSVVGWAAVRERMQQVEPAADGVGAVSRDLEKYGALTSSNTAANNADCAWVEA P16: GGKWSKWSVSGWPAIRERMRPIEPAADGVGAVSRDLARHGAVTSSNTAATNAACAWLEA P17: GGKWSKSRVVGWAAVRERMRQIEPAADGVGAVSRDLGRHGAVTSSNTAANNAACAWLEA P18: GGKWSKTRVIGWSAVRERMRQIEPAADGVGAVSRDLGKYGAVTSRNTAANNAACARLEA P19: GGKWSKSRVVGWPTIRERMRRTEPAADGVGAVSRDLDRYGALTSSNTAATNADCAWVEA P20: GGKWSKWSVIGWPTIRERMRQVEPAADGVGAVSRDLERYGALTSSNTATTNAACAWLEA P21: GGKWSKRSVGGWAA IRERMRPAEPAADGVGAVSRDLDRYGALTSSNPAATNADCAWLEA P22: GGKWSKSRVVGWAAVRERMRQIEPAADGVGAVSRDLGRHGALTSSNTAANNAACAWLEA P23: GGKWSKTRVGGWPTIRERIQRAEPTADGVGAVSRDLGKYGAVTSSNTATNNAACAWVEA P2 4: GGKWSKSRVVGWAAVRERMRQIEPAADGVGAVSRDLGRHGAVTSSNTAANNAACAWLEA P2 5: GGKWSKWRVGGWPAIRERIKPIEPAADGVGAVSRDLGRHGAITSSNTPANNAACAWLEA P27: GGKWSKWSVGGWAAVRERMRRAEPAADGVGAVSRDLEKHGAITSSNTPANNAACAWLEA P28: GGKWSKSRVVGWSAVRERMRPAEPAADGVGAVSRDLGRHGALTSSNTATNNADCAWLEA P29: GGKWSKSRVGGWAAIRERIRPTEPAADGVGAVSRDLARHGALTSSNTAANNAACAWVEA P30: GGKWSKSRVGGWSAVRERMKQVEPAADGVGAVSRDLERHGAITSSNTPANNAACAWLEA

Trial\#3

P31: GGKWSKPSVSGWAAVRERIRRAEPAADGVGAVSRDLGKHGALTSSNTATNNAACAWLEA P32: GGKWSKWSVSGWPTIRERMRRAEPAADGVGAVSRDLARHGAVTNSNTAANNAACAWVEA P34: GGKWSKSRVIGWSTIRERMRPAEPAANGVGAVSRDLAKHGAVTSSNTATNNADCAWLEA P35: GGKWSKWRVGGWSAIRERMQRAEPAADGVGAVSRDLARHGAVTSSNTAANNAACAWVEA

Trial\# 4

P2: GGKWSKPRVSGWPTIRERMRPAEPAADGVGAVSRDLEKHGAITSSNTAANNAACAWLEA P3: GGKWSKSRVSGWPTVRERIKRAEPAADGVGAVSRDLGKYGAVTSSNTATTNTDCAWVEA P4: GGKWSKWRVGGWAAVRERMRQAEPAADGVGAVSRELGRYGAIRSSNTPTTNAACAWLEA P8: GGKWSKWRVGGWPTVRERMRPVEPAADGVGAVSRDLARYGAITSSNTPATNADCAWLEA P9: GGKWSKPSVSRWSAVRERMRPVEPAADGVGAVSRDLGKYGARTSSNTPTNNAACAWLEA P12: GGKWSKSSVIGWSAIRERMRRTEPAANGVGAVSRDLGRYGAVTSNNTATTNAACAWLEA P15: GGKWSKSRVGGWAA IRERMQRAEPAADGVGAVSRDLGRYGAVTSSNTAATNAACAWLKA P16: GGKWSKWRVGGWPAIRERMQPVEPAADGVGAVSRDLDRYGALTSSNTPANNAACAWLEA P18: GGKWSKPRVSGWPTIRERMRPAEPAADGVGAVSRDLEKHGAITSSNTAANNAACAWLEA

\section{Nef mutants selected for binding to actin}

Trial\# 1

A1: GGKWSKWRVSGWSAVRERIRQAEPAADGVGAVSRDLAKHGALTSSNTPTNNADCAWLEA

A2: GGKWSKWRVGGWAAIRERMRPVEPAADGVGAVSRDLEKHGALTSSNTAANNADCAWLEA

A3: GGKWSKWSVSGWPAVRERMRPAEPAADGVGAVSRDLGRYGAITSSNTATNNADCAWVEA

A4: GGKWSKPRVGGWPTVRERIQRAEPAADGVGAVSRDLGKHGAITSSNTPTTNAACAWIEA

A5: GGKWSKWRVGGWAAVRERMRRAEPAADGVGAVSRDLGRYGALTSSNTPTTNAACAWLEA 
A6: GGKWSKPRVGGWPTIRERIRPAEPAADGVGAVSRDLERHGALTSSNTPATNAACAWLEA A 7: GGKWSKWSVGGWAAIRERMRPIEPAADGVGAVSRDLAKHGAVTSSNTPTTNADCAWLEA A8: GGKWSKWSVGGWPTIRERLGRAEPAADGVGAVSRDLDRHGAVTSSNTAATNAACAWLEA A11: GGKWSKSRVGGWSAIRERMRPAEPAADGVGAVSRDLEKYGAVTSSNTATNNADCAWVEA A13: GGKWSKWSVIGWPAIRERIRRAEPAADGVGAVSRDLGKYGAVTSSNTAATNADCAWVEA A14: GGKWSKWRVGGWSA IRERMQPAEPAADGVGAVSRDLGRYGALTSSNTAANNAACAWGEA A15: GGKWSKWSVGGWSAVRERMRRAEPAADGVGAVSRDLGRHGALTSSNTAATNAACAWLEA A16: GGKWSKWSVGGWSAVRERMRQVEPAADGVGAVSRDLGKYGALTSSNTATTNAACAWLEA A2 0 : GGKWSKPSVVGWAAIRERMRRTEPAADGVGAVSRDLARHGALTSSNTAATNAACAWLEA A22: GGKWSKWSVVGWPTIRERMRRAEPAADGVGAVSRDLGRYGAVTSSNTATTNAACAWLEA A2 3: GGKWSKWRVGGWSA IRERMQRAEPAADGVGAVSRDLAKYGAITSSNTAATNADCAWLEA A27: GGKWSKWSVSGWPAIRERMRRAEPAADGVGAVSRDLARHGAITSSNTATNNAACAWLEA A28: GGKWSKWRVGGWSTVRERMQRTEPAADGVGAVSRDLDRHGAITSSNTATNNADCAWVEA A29: GGKWSKWSVGGWPAIRERMRRAEPAADGVGAVSRDLGKYGAITSSNTAANNAACAWLEA A30 : GGKWSKWSVGGWAAIRERIRRVEPAADGVGAVSRDLGRYGAVTSSNTAANNADCAWVEA A32: GGKWSKWSVGGWPTIRERMRQTEPAADGVGAVSRDLDKYGAITSSNTAANNAACAWVEA A33: GGKWSKPRVGGWSAIRERMRPVEPAADGVGAVSRDLDKYGAVTSSNTAANNAACAWVEA A37: GGKWSKCSVGGWPAIRERMRRIEPAADGVGAVSRDLEKHGALTSSNTPTNNAACAWVEA A38: GGKWSKWSVGGWAAVRERMKRTEPAADGVGAVSRDLGRYGAITSSNTATNNAACAWIEA A39: GGKWSKWSVGGWAAIRERIRQAEPAADGVGAVSRDLDKHGAITSSNTATTNAACAWWEA A 40 : GGKWSKWRVGGWPAVRERIKQAEPAADGVGAVSRDLGRYGAITSSNTATTNADCAWVEA A 41 : GGKWSKWRVGGWPTVRERMQQVEPAADGVGAVSRDLDRYGAITSSNTAANNADCAWVEA A 42: GGKWSKWSVGGWAAIRERMRPIEPAADGVGAVSRDLAKHGAVTSSNTPTTNADCAWLEA A 43: GGKWSKWSVGGWSTIRERIRRAEPAADGVGAVSRDLARYGAVTSSNTPTTNADCAWVEA A 4 : GGKWSKPSVVGWSTVRERIRRTEPAADGVGAVSRDLGRHGAVTSSNTAANNAACAWLEA

Trial\#2

A1: GGKWSKPRVVGWSAIRERMRPVEPAADGVGAVSRDLGRHGAVTSSNTAATNADCAWVEA A2: GGKWSKWSVIGWPTIRERMRQTEPAADGVGAVSRDLARYGALTSSNTAANNADCAWVEA A3: GGKWSKWSVVGWPTIRDRMRPIEPAADGVGAVSRDLGRYGAVTSSNTAANNAACAWVEA A4: GGKWSKWSVIGWPTIRERMRQTEPAADGVGAVSRDLARYGAVTSSNTAANNADCAWVEA A5: GGKWSKWSVIGWPTIRERMRQTEPAADGVGAVSRDLARYGALTSSNTAANNADCAWVEA A 6: GGKWSKWSVIGWPTIRERMRQTEPAADGVGAVSRDLARYGALTSSNTAANNADCAWVEA A7: GGKWSKWRVGGWPAVRERMRPAEPAADGVGAVSRDLGRHGAITSSNTPTNNAACAWLEA A8: GGKWSKPSVGGWATIRERMRPIEPAADGVGALSRDLGRYGAVTSSNTATTNAACAWLEA A10: GGKWSKWSVIGWPTIRERMKQTEPAADGVGALSRDLARYGALTSSNTAANNADCAWVEA A11: GGKWSKWSVIGWPTIRERMRQTEPAADGVGAVSRDLARYGALTSSNTAANNADCAWVEA A12: GGKWSKWSVIGWPTIRERMRQTEPAADGVGAVSRDLARYGALTSSNTAANNADCAWVEA A13: GGKWSKWSVIGWPTIRERMGQTEPAPDGVGALSRDLARYGALTSSNTAANNADCAWVEA A15: GGKWSKCSVGGWPTIRERIRRAEPAADEVGAVSRDLGRYGAITSSNTPATNAACAWVEA A16: GGKWSKWSVIGWPTIRERMRQTEPAADGVGAVSRDLARYGALTSSNTAANNADCAWVEA A18: GGKWSKWSVIGWPTIRERMRQTEPAADGVGAVSRDLGRYGALTSSNTAANNADCAWVEA A19: GGKWSKWSVIGWPTIRERMRQTDPAADGVGALSRDLARYGALTSSNTAANNADCAWVEA A20: GGKWSKWSVIGWPTIRERMRQTEPAADGVGAVSRDLARYGALTSSNTAANNADCAWVEA A21: GGKWSKWSVSGWPAIRERMRPVEPAADGVGAVSRDLEKHGAVTSSNTAANNADCAWVEA A22: GGKWSKWRVGGWSAVRERIQRTDPAADGVGALSRDLAKYGAITSSNTPANNAACAWLEA A24: GGKWSKPRVGGWAAVRERMKQIEPAADGVGALSRDLGRYGAITSSNTAANNAACAWLEA A25: GGKWSKWSVIGWPTIRERMRQTEPAAHGVGALSRDLARYGALTMSNTAANTADCAWVEA A26: GGKWSKWSVIGWPTIRERMRQTDPAPDGVGAVSRDLARYGALTSSNTAANNADCAWVEA

Trial\#3

A28: GGKWPKWSVIGWPTIRERMRQTEPAADGVGAVSRDLARYGALTSSNTAANNADCAWVEA A30: GGKWSKWSVIGWPTIRERMRQTEPAADGVGAVSRDLARYGALTSSNTAANNADCAWVEA A31: GGKWSKWSVSGWPTVRERMRRTEPAADGVGAVSRDLAKYGAVTSSNTTANNAACAWLEA A32: GGKWSKWSVIGWPTIRERMRQTEPAADGVGAVSRDLARYGALTSSNTAANNADCAWVEA A33: GGKWSKWSVIGWPTIRERMRQTEPAADGVGAVSRDLARYGALTSSNTAANNADCAWVEA A35: GGKWSKWSVIGWPTIRERMRQTEPAADGVGAVSRDLARYGALTSSNTAANNADCAWVEA A36: GGKWSKWSVIGWPTIRERMRQTEPAADGVGAVSRDLARYGALTSSNTAANNADCAWVEA A39: GGKWSKCSVGGWPTIRERIRRAEPAPDEVGAVSRDLGRYGAITSSNTPATNAACAWVEA A40: GGKWSKWSVGGWPTIRERIRRADPAADEVGAVSRDLGRYGAITSSNTPATNAACAWVEA 
A 46: GGKWSKWSVIGWPTIRERMRQTEPAADGVGAVSRDLARYGALTSSNTAANNAACAWVEA

A47: GGKWSKCSVGGWPTIRERIRRAEPAADEVGAVSRDLGRYGAITSSNTPATNAACAWVEA

A51: GGKWSPWSVIGWPTIRERMRQTEPAADGVGAVSRDLARYGALTSSNTAANNADCAWVEA

A63: GGKWSKWSVIGWPTIRERMRQTDPAADGVGAVSRDLARYGALTSSNTAANNADCAWVEA

A64: GGKWSKWSVIGWPTIRERMRQTDPAADGVGAVSRDLARYGALTSSNTAANNADCAWVEA

A76: GGKWSKWSVIGWPTIRERMRQTEPAADGVGAVSRDLARYGALTSSNTAANNADCAWVEA

A79: GGKCSQWSVIGWPTIRERMRQTEPAADGVGAVSRDLARYGALTSSNTAANNADCAWVEA

A80: GGKWSKWSVIGWPTIRERMRQTEPAADGVGAVSRDLARYGALTSSNTAANNADCAWVEA

A82: GGKWSKWSVSGWPAVRERMRPIEPAADGVGAVSRDLAKYGAVTSSNTPANNADCAWVEA

Nef mutants selected for binding to $p 56^{\text {lck }}$

Trial\# 1

L1: GGKWSKPRVSGWAAVRERIRRAEPAADGVGAVSRDLGKYGAITSSNTPANNADCAWLEA

L2: GGKWSKTRVGGWSAIRERMKRAEPAADGVGAVSRDLDRYGALTSSNTAATNADCAWVEA

L3: GGKWSKWRVGGWAAIRERMKQTEPAADGVGAVSRDLGRYGAITSSNTPTNNADCAWVEA

L4: GGKWSKWSVSGWPTVRERIRRAEPAADGVGAVSRDLQRYGAVTSSNTATNNAACAWLEA

L5: GGKWSKPRVSGWSAIRERMRPVEPAADGVGAVSRDLARYGAITSSNTATNNAACAWLEA

L6: GGKWSKPRVSGWSAIRERMRPTEPAADGVGAVSRDLARYGAITSSNTATNNAACAWVEA

L7: GGKWSKPRVSGWSTIRERIRPVEPAADGVGAVSRDLQRYGAVTSSNTAANNADCAWLEA

L8: GGKWSKWRVGGWPAVRERIQPTEPAADGVGAVSRDLGRYGAITSSNTAATNADCAWLEA

L9: GGKWSKPRVGGWSTIRERMRQVEPAADGVGAVSRDLDKYGAVTSSNTAATNAACAWLEA

L11: GGKWSKTSVSGWAAVRERMRRTEPAADGVGAVSRDLGRHGAVTSSNTAANNAACAWLEA

L12: GGKWSKWSVGGWAAIRERIRRAEPAADGVGAVSRDLARHGAITSSNTAANNADCAWVEA L13: GGKWSKRSVGGWAAIRERIRQAEPAADGVGAVSRDLGRYGAITSSNTPANNADCAWVEA L14: GGKWSKWSVGGWPTIRERIRRTEPAADGVGAVSRDLEKYGAVTSSNTATNNAACAWVEA L17: GGKWSKWRVGGWPAVRERMRPVEPAADGVGAVSRDLGKHGALTSSNTPTTNADCAWVEA L18: GGKWSKWSVSGWSAVRERMRPAEPAADGVGAVSRDLAKHGALTSSNTAATNAACAWLEA L19: GGKWSKWSVGGWPTIRERMRRTEPAADGVGAVSRDLGRYGALTSSNTAATNADCAWVEA L20: GGKWSKTRVIGWPTIRERMRRAEPAADGVGAVSRDLGKYGAVTSSNTPTNNAACAWVEA L21: GGKWSKRRVIGWPTIRERIRQ IEPAADGVGAVSRDLGKIWSTHKCNTATTNAAYAWLEA L22: GGKWSKPRVSGWAAIRERMRPTEPAADGVGAVSRDLARYGAVTSSNTATNNADCAWLKA L25: GGKWSKWSVGGWAAVRERMRRTEPAADGVGAVSRDLAKHGALTSSNTPANNAACAWLKA L26: GGKWSKWSVIGWPTIRERMRRTEPAADGVGAVSRDLAKHGAITSSNTPANNADCAWLKA L27: GGKWSKPSVIGWSAIRERMKRVEPAADGVGAVSRDLGRYGAVTSSNTATNNADCAWVKA L33: GGKWSKWRVSGWSAIRERMRPAEPAADGVGAVSRDLARYGALTSSNTPANNAACAWLKA L34: GGKWSKPRVVGWAAVRERMKRAEPAADGVGAVSRDLAKHGALTS SNTAANNAACAWLKA

\section{References}

(1) Olszewski, A.; Sato, K.; Aron, Z. D.; Cohen, F.; Harris, A.; McDougall, B. R.; Robinson, W. E., Jr.; Overman, L. E.; Weiss, G. A., Proc. Natl. Acad. Sci. USA 2004, 101, (39), 14079-14084.

(2) Bewley, C. A.; Ray, S.; Cohen, F.; Collins, S. K.; Overman, L. E. J. Nat. Prod. 67, 1319 (2004).

(3) Cohen, F.; Overman, L. E. J. Amer. Chem. Soc. 123, 10782 (2001).

(4) Cohen, F.; Overman, L. E.; Ly Sakata, S. K. Org. Lett. 1, 2169 (1999).

(5) Aron, Z. D.; Pietraszkiewicz, H.; Overman, L. E.; Valeriote, F.; Cuevas, C. Bioorg. Med. Chem. Lett. 14, 3445 (2004).

(6) Coffey, D. S.; McDonald, A. I.; Overman, L. E.; Rabinowitz, M. H.; Renhowe, P. A. J. Amer. Chem. Soc. 122, 4893 (2000).

(7) Sidhu, S. S.; Weiss, G. A. in Phage Display: A Practical Approach H. L. Lowman, \& Clackson, T., Ed. (Oxford University Press, 2004) pp. 27-41. 